\title{
PROCESO PARA ESTIMAR EL NIVEL REAL DE CONCIENCIA DE LOS USUARIOS DE FACEBOOK RESPECTO A PRIVACIDAD Y SEGURIDAD EN LA RED SOCIAL
}

\author{
Ramiro Rios-Paredes ${ }^{1,2}$ y Diego Rios-Salgado ${ }^{3}$ \\ Universidad de las Fuerzas Armadas - ESPE, Ecuador. rarios@espe.edu.ec \\ 2 Facultad de Informática Universidad Nacional de La Plata - UNLP, Argentina. ramiro.riosp@info.unlp.edu.ar \\ ${ }^{3}$ Faculté de Sciences de l'Université de Reims Champagne-Ardenne, France. diego.rios-salgado@etudiant.univ-reims.fr
}

\begin{abstract}
Resumen. Se propone un proceso para estimar el nivel real de conciencia sobre privacidad y seguridad de los usuarios de Facebook, fundamentado en dos factores determinantes: el nivel de riesgo generado por los comportamientos psicosociales en Facebook; y, el nivel de cultura de seguridad y privacidad del usuario. Al aplicar el proceso a una muestra de usuarios de Facebook, y comparar el nivel de conciencia real con el nivel de conciencia hipotético supuesto por ellos, se han obtenido tres opciones: $41.15 \%$ tienen nivel hipotético $=$ nivel real; $20.35 \%$ revelan nivel hipotético < nivel real; y, $38.50 \%$ demuestran nivel hipotético > nivel real. Conclusiones: 1) La aplicación iterativa conjunta de los lineamientos de la Teoría Fundamentada y la Hermenéutica, permitieron estructurar una sólida fundamentación, concretada en un modelo conceptual de causas de los ciber-riesgos. 2) El proceso propuesto refleja con fidelidad la realidad del usuario de Facebook, con tres opciones: nivel hipotético coincide con el nivel real; nivel hipotético representa que se subestima o que se sobrestima con respecto al real que posee. 3) El proceso desarrollado provee datos complementarios que contribuyen para un desenvolvimiento más seguro en la red social.
\end{abstract}

Palabras clave: Ciber-Riesgos; Comportamientos Psicosociales; Cultura de Seguridad y Privacidad; Facebook; Nivel de Conciencia.

\section{A PROCESS TO ESTIMATE THE ACTUAL LEVEL OF AWARENESS OF FACEBOOK USERS REGARDING PRIVACY AND SECURITY WITHIN THE SOCIAL NETWORK}

\begin{abstract}
We propose a process to estimate the actual level of privacy and security of Facebook users. It was based upon two factors: the risk level, which was generated by psychosocial behaviors on Facebook; and the user's level of culture in terms of security and privacy. This process was applied to a sample of Facebook users and, when comparing their level of actual awareness, related to security and privacy, with their level of hypothetical awareness (the one presumed by them); three options were obtained: hypothetical level $=$ actual level, for the $41.15 \%$ of interviewees; hypothetical level < actual level for the $20.35 \%$; and, hypothetical level > actual level for the $38.50 \%$. Conclusions: 1) The iterative application of the guidelines, provided by the Grounded Theory and Hermeneutics, allowed to structure a solid foundation for a conceptual model of causes of cyber risks. 2) The process authentically reflects the reality of the Facebook users in three ways: they can have a hypothetical level that coincides with their actual level; or a hypothetical level which may be underestimated or overestimated when compared to their actual level. 3) The process provides with complementary data which may contribute to a safer experience within the social network.
\end{abstract}

Keywords: Cyber risks; Security, Privacy; Facebook; Level of Awareness; Psychosocial Behaviors.

\section{INTRODUCCIÓN}

Este estudio, perteneciente al área de Computación Social, fue motivado por los siguientes sucesos trascendentes: 1) El uso cada vez mayor de la red social Facebook. 2) Lo afirmado 
en Wallbridge: "Aunque en muchos sentidos un usuario ofrece "consentimiento" cuando se registra en un sitio en línea, la mayoría desconoce las implicaciones de proporcionar voluntariamente información personal en los perfiles, además de no estar al tanto de cómo se puede estar procesando esta información" (2009, p. 88). 3) Aportar en la reducción de la deficiencia que se afirma en UIT: "Hay relativamente pocos datos recogidos sobre las repercusiones del acceso a las TIC y de su utilización individual y en el hogar, y también son pocos los trabajos analíticos sobre este tema" (2014, p. 80).

El proceso, que se describe en las siguientes secciones de este documento, requirió de: A) Una fundamentación teórica, resultado de un proceso de investigación documental cualitativo, que se consolidó en un modelo conceptual, con enfoque interdisciplinario, de las causas de los ciber-riesgos en el entorno de Facebook; $y$, en las características significativas que conforman los factores determinantes del grado de conciencia sobre privacidad y seguridad. B) Una entrevista estructurada desarrollada para recabar información de los usuarios de Facebook respecto a sus comportamientos en línea; y, a conocimientos varios sobre privacidad y seguridad en la red social, configuraciones y políticas de datos que se manejan en Facebook. C) La concepción de un proceso, desarrollado con perspectivas cuantitativas, para estimar el grado real de conciencia sobre privacidad y seguridad de cada usuario de Facebook entrevistado.

\section{PROPÓSITO DEL ESTUDIO}

\subsection{Objetivo}

Estructurar un proceso para la estimación del nivel real de conciencia de los usuarios de Facebook respecto a privacidad y seguridad en la red social.

\subsection{Hipótesis}

La estimación del nivel real de conciencia de usuarios de Facebook respecto a privacidad y seguridad en la red social puede seguir un proceso basado en los comportamientos psicosociales y la cultura de privacidad y seguridad que las personas manifiestan en sus interacciones virtuales en Facebook. 


\subsection{Pregunta de Investigación}

¿Es posible estructurar un proceso para estimar el nivel real de conciencia de los usuarios de Facebook respecto a privacidad y seguridad en la red social que se fundamente en los comportamientos psicosociales y en la cultura de privacidad y seguridad que las personas manifiestan en sus interacciones virtuales en Facebook?

\subsection{Contexto de Investigación}

Tomando en cuenta el objetivo y la hipótesis planteados, el contexto de investigación se dividió en dos partes: A) el contexto de investigación para estructurar la fundamentación de la propuesta, conformado por publicaciones indexadas, difundidas por Internet, relacionadas con los tres tipos de ciber-riesgos en el entorno de la red social Facebook, descritos en (Instituto de Auditores Internos de España, 2016) y (Me Conecto Sin Clavos, 2017), a saber: 1) Contra la información; 2) Contra el ser humano; y, 3) Contra la infraestructura. Y, B) el contexto de investigación para instrumentar la propuesta, y hacer una primera aproximación hacia su validación mediante la verificación de la fidelidad con que refleja la realidad, constituido por una muestra no representativa del universo de usuarios de Facebook, compuesta por 226 miembros del entorno de influencia del investigador, hombres y mujeres ecuatorianos con distintos niveles de formación académica, pertenecientes a los grupos etarios: adultos mayores (edad: mayores a 64 años), adultos (edad: entre 41 y 64 años), adultos jóvenes (edad: en el rango de 18 a 40 años) y niños/adolescentes (edad: menor a 18 años), como se muestra en la Figura 1.

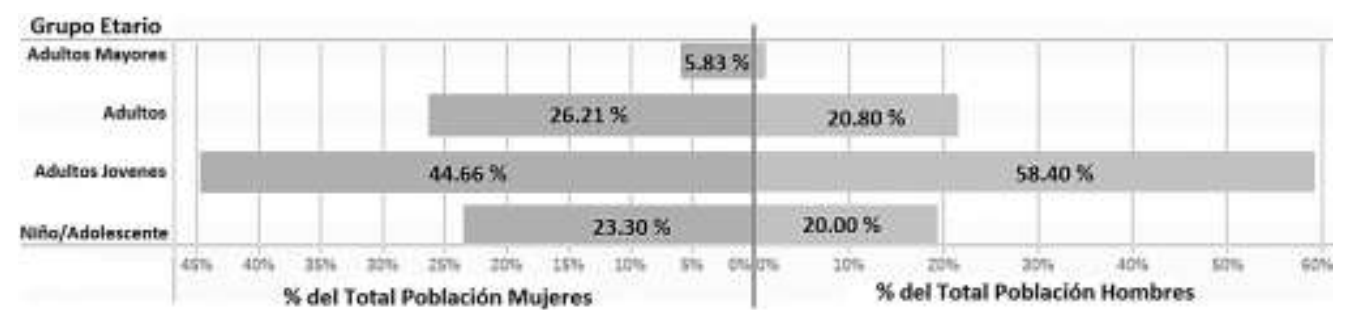

Figura 1. Pirámide de población por grupo etario de usuarios de Facebook entrevistados

\section{MARCO METODOLÓGICO}

\subsection{Para la Concreción de la Fundamentación}

La investigación documental utilizó un enfoque de naturaleza inductiva. Se aplicó como técnica de recolección de datos: heurística en la selección de publicaciones útiles a la investigación. Para la interpretación documental, se empleó la hermenéutica, desarrollando 
el análisis cualitativo de los datos con apoyo del computador, mediante el software Atlas Ti; logrando un acercamiento progresivo a la estructuración de las causas de los ciber-riesgos en la red social mediante la aplicación de los lineamientos dados por la Teoría Fundamentada: ejecutar iterativamente la organización de los datos en códigos, conceptos y categorías, para finalmente concretar los términos descritos en la Tabla 1.

Tabla 1. Conceptos y categorías en que se estructuraron las causas de los ciber-riesgos en el entorno de Facebook de acuerdo a los lineamientos de la Teoría Fundamentada

\begin{tabular}{ccc}
\hline $\begin{array}{c}\text { Números de } \\
\text { identificación }\end{array}$ & $\begin{array}{c}\text { Nombres de identificación de los conceptos que } \\
\text { agrupan las causas de los ciber-riesgos en Facebook }\end{array}$ & $\begin{array}{c}\text { Nombres de identificación } \\
\text { de las Categorías } \\
\text { (Disciplinas Científicas) }\end{array}$ \\
\hline 1 & $\begin{array}{c}\text { Vulnerabilidades atribuibles a los usuarios: a) Nivel de } \\
\text { cultura de seguridad y privacidad; y, b) Nivel de riesgo } \\
\text { por comportamientos psicosociales. }\end{array}$ & Ciencias Psicosociales \\
\hline 2 & Almacenamiento de grandes volúmenes de información & \\
3 & Facilidades que ofrece el servicio & Ciencias Informáticas \\
5 & Vulnerabilidades de la plataforma tecnológica & \\
\hline 6 & Desarrollos tecnológicos & Ciencias Jurídicas \\
\hline
\end{tabular}

La Tabla 2 sintetiza las publicaciones que dan soporte al compendio de la Tabla 1.

Tabla 2. Publicaciones seleccionadas que soportan los conceptos en que se organizaron las causas de los ciber-riesgos en el entorno de Facebook.

\begin{tabular}{|c|c|c|c|c|c|c|}
\hline \multirow[t]{2}{*}{ Referencia a la publicación } & \multicolumn{6}{|c|}{$\begin{array}{c}\text { Número de identificación de los conceptos que agrupan las } \\
\text { causas de los ciber-riesgos en Facebook }\end{array}$} \\
\hline & 1 & 2 & 3 & 4 & 5 & 6 \\
\hline (UNESCO, 2012) & & YES & & YES & YES & \\
\hline (Algarni, Xu, \& Chan, 2014) & YES & YES & YES & YES & & \\
\hline $\begin{array}{l}\text { (Netter, Herbst, \& Pernul, } \\
\text { 2013) }\end{array}$ & YES & YES & YES & YES & & YES \\
\hline (Cornejo \& Tapia, 2011) & & YES & YES & & & \\
\hline $\begin{array}{l}\text { (Lara, Pincheira, \& Vera, } \\
\text { 2014) }\end{array}$ & & & & & & YES \\
\hline (OECD, 1980) & & YES & & YES & YES & YES \\
\hline (OECD, 2011) & & YES & & YES & YES & \\
\hline (Wong, 2014) & YES & YES & & YES & YES & \\
\hline $\begin{array}{l}\text { (Zhang, Sun, Zhu, \& Fang, } \\
\text { 2010) }\end{array}$ & & & & & YES & \\
\hline (Zhan \& Fang, 2011) & YES & YES & YES & YES & & \\
\hline
\end{tabular}




\subsection{Para Instrumentar el Proceso de Estimación}

La estructuración del proceso se basó en una metodología esencialmente cuantitativa, cuyo punto de partida fue la fundamentación y continuó con las etapas que se muestran en la Figura 2.

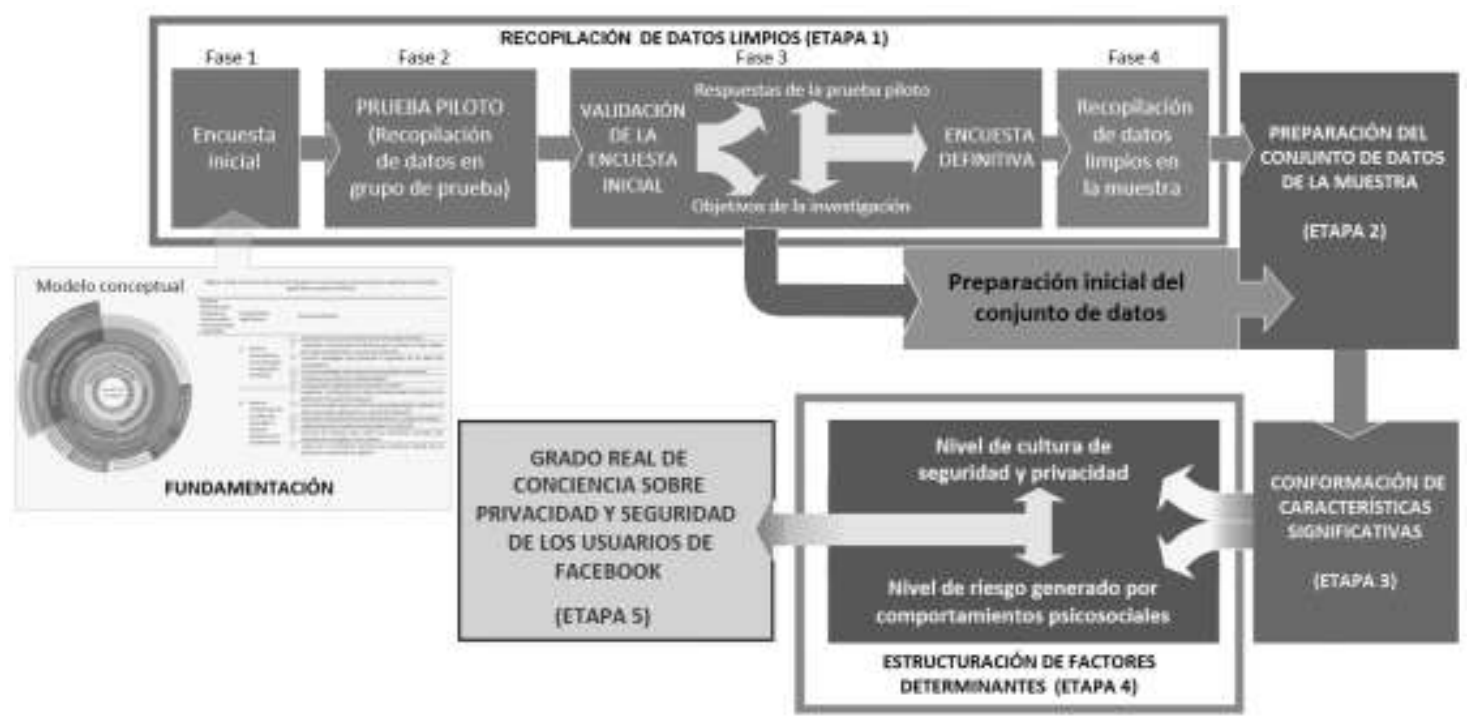

Figura 2. Proceso de concepción de la propuesta de estimación

La etapa 1: recopilación de datos limpios, ejecutó una limpieza proactiva de datos, conformada de cuatro fases. En la fase 1 se elaboró una entrevista estructurada inicial, dedicada a recopilar datos relacionados con diferentes aspectos dados en la fundamentación del estudio: sociodemográficos del entrevistado, de frecuencia de uso de Facebook, de actitudes en la interactividad virtual, de configuraciones de privacidad/seguridad, de conocimiento de los fundamentos de privacidad/seguridad, de comprensión de la política de datos de Facebook, de aspectos legales elementales aplicables en el entorno de Facebook. Como fase 2, se aplicó la entrevista estructurada inicial a un reducido grupo de prueba de 30 personas (PRUEBA PILOTO). En la fase 3, se perfeccionó la entrevista estructurada y se acordó: 1) Para enfocar la visión interdisciplinaria del modelo conceptual de la Figura 3, la entrevista estructurada final estaría conformada por las secciones indicadas en la Tabla 3. 2). Una sola pregunta en la sección de datos sociodemográficos, era suficiente para determinar el nivel hipotético de conciencia sobre privacidad de los usuarios de Facebook: 
“¿Cómo calificaría su nivel de conocimientos sobre seguridad y privacidad en la red social Facebook?", las opciones de respuesta fueron: Avanzado, Medio, Básico y Ninguno; nivel hipotético que apoyaría posteriormente la validación de la propuesta. 3) Las preguntas de las secciones 2 a la 7 servirían para determinar el nivel real de conciencia sobre privacidad y seguridad de los usuarios de la red social, de conformidad a las características significativas señaladas en la Tabla 4. 4) Condicionar al entrevistado a responder todas las preguntas. Y, 5) La entrevista estructurada definitiva se distribuiría a la muestra seleccionada mediante correo electrónico. La fase 4, última de la primera etapa, consistió en aplicar la entrevista estructurada definitiva a la muestra seleccionada de usuarios de Facebook.

Tabla 3. Secciones de la entrevista estructurada

\begin{tabular}{ccc}
\hline \multirow{2}{*}{ Ordinal } & Nombres de las secciones & $\begin{array}{c}\text { Número } \\
\text { de } \\
\text { preguntas }\end{array}$ \\
\hline 1 & Datos socio-demográficos del encuestado & 9 \\
\hline 2 & Uso de Facebook & 9 \\
\hline 3 & Conocimiento de las configuraciones de privacidad en Facebook & 5 \\
\hline 4 & Conocimiento de los principios de seguridad y privacidad en Facebook & 5 \\
\hline 5 & Comprensión de la política de privacidad y la política de datos de & 8 \\
\hline 6 & $\begin{array}{c}\text { Conocebook } \\
\text { contexto operativo; y, de seguridad y privacidad en Facebook }\end{array}$ & 5 \\
\hline 7 & Comportamientos psicosociales & 13 \\
\hline
\end{tabular}

Paralelamente a la fase tres de la etapa 1, se desempeñó la preparación inicial del conjunto de datos, en la cual se establecieron las siguientes reglas: 1) Cada usuario de Facebook entrevistado sería representado por una serie de atributos; 2) cada atributo tendría un valor, según la respuesta a una o varias preguntas vinculadas de la entrevista estructurada definitiva, de tal forma que al final, cada usuario se simbolizaría por una lista de números contenida en un vector de atributos; 3) se determinó que el número de atributos para caracterizar a cada persona entrevistada sería 32 (valor obtenido tomando en cuenta que el número de preguntas de la encuesta eran 54, y que algunas se integraban para dar un atributo), por lo tanto, cada persona entrevistada sería un punto en un espacio multidimensional de 32 dimensiones. La etapa 2 corresponde a la aplicación de las reglas generadas en la preparación inicial del conjunto de datos, al conjunto de datos de la muestra.

En las etapas 3 y 4 , y siguiendo el detalle de la Tabla 4, se aplican secuencialmente las siguientes reglas: 1) Para la conformación de las características significativas, para cada 
usuario de Facebook, se calcula la media aritmética de los valores de los atributos que conforman la respectiva característica. 2) El promedio de los valores obtenidos para las características que conforman la cultura de seguridad y privacidad en línea del usuario de Facebook determinarían su nivel de cultura. 3) El nivel de riesgo generado por los comportamientos psicosociales se estima de forma similar a lo señalado en la regla 2.

En la etapa 5, se aplican dos reglas: 1) La media aritmética de los valores de los factores determinantes: cultura de seguridad y privacidad en línea del usuario de Facebook, y nivel de riesgo generado por sus comportamientos psicosociales, determina un valor para el nivel real de conciencia sobre privacidad y seguridad en cada usuario de Facebook. 2) El valor calculado con la regla anterior, se encasilla en uno de los 4 posibles valores cualitativos de nivel real de conciencia sobre privacidad y seguridad en cada usuario de Facebook: Avanzado, Medio, Básico, Ninguno, definidos por los siguientes rangos de valores: Avanzado: [0.76,1]; Medio: [0.51,0.75]; Básico: [0.26,0.50]; Ninguno: [00.0,0.25].

\section{RESULTADOS}

\subsection{Fundamentación de la Propuesta}

\subsubsection{Modelo Conceptual de las Causas de los Ciber-riesgos en el Entorno de Facebook}

Las causas de los ciber-riesgos en el entorno de Facebook fueron organizadas en un modelo conceptual, que resultó mejor estructurado con un enfoque interdisciplinario desde las ciencias: Psicosociales, Jurídicas, e Informáticas, como se muestra en la Figura 3.

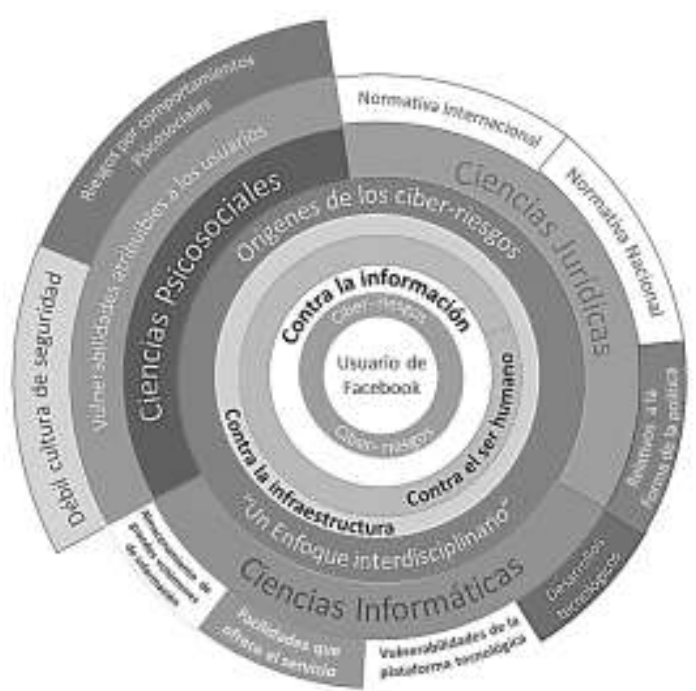

Figura 3. Modelo conceptual, con enfoque interdisciplinario, de las causas de los ciber-riesgos en el entorno de Facebook 


\subsubsection{Factores Determinantes del Grado de Conciencia sobre Privacidad y Seguridad}

Las vulnerabilidades atribuibles a los usuarios de Facebook, presentes en el modelo conceptual de la Figura 3, se consideraron en este estudio como los factores determinantes del grado de conciencia sobre privacidad y seguridad; $y$, están definidos por las características significativas y los atributos presentes en la Tabla 4.

Tabla 4. Factores determinantes del grado de conciencia sobre privacidad y seguridad, características significativas y grupos de atributos.

\begin{tabular}{|c|c|c|}
\hline $\begin{array}{c}\text { Factores } \\
\text { determinantes } \\
\text { del grado de } \\
\text { conciencia sobre } \\
\text { privacidad y } \\
\text { seguridad }\end{array}$ & $\begin{array}{l}\text { Características } \\
\text { significativas }\end{array}$ & Grupos de atributos \\
\hline \multirow{17}{*}{$\begin{array}{l}\text { Nivel de cultura } \\
\text { de seguridad y } \\
\text { privacidad }\end{array}$} & \multirow{6}{*}{$\begin{array}{l}\text { A. Nivel de } \\
\text { conocimientos } \\
\text { sobre } \\
\text { principios de } \\
\text { seguridad y } \\
\text { privacidad. }\end{array}$} & 1. ¿Reconoce lo que es una violación de su Privacidad en línea? \\
\hline & & $\begin{array}{c}\text { 2. ¿ldentifica correctamente los términos que se refieren a ciber- } \\
\text { riesgos a los que está expuesto un usuario de Facebook? }\end{array}$ \\
\hline & & $\begin{array}{l}\text { 3. ¿Conoce estrategias para preservar la seguridad de los datos } \\
\text { del computador? }\end{array}$ \\
\hline & & $\begin{array}{l}\text { 4. ¿Conoce estrategias para preservar la privacidad en } \\
\text { Facebook? }\end{array}$ \\
\hline & & 5. ¿Entiende el principio de confidencialidad? \\
\hline & & 6. ¿Comprende el significado de privacidad en línea? \\
\hline & \multirow{6}{*}{$\begin{array}{l}\text { B. Nivel de } \\
\text { comprensión } \\
\text { de la política } \\
\text { de privacidad y } \\
\text { datos de } \\
\text { Facebook y } \\
\text { sus } \\
\text { actualizacione } \\
\text { s. }\end{array}$} & $\begin{array}{l}\text { 1. ¿Identifica correctamente los temas fundamentales enfocados } \\
\text { en la política de Privacidad de Facebook? }\end{array}$ \\
\hline & & $\begin{array}{c}\text { 2. ¿Conoce las aplicaciones o personas que pueden llegar a } \\
\text { obtener sus datos personales registrados en su perfil de } \\
\text { Facebook? }\end{array}$ \\
\hline & & $\begin{array}{l}\text { 3. ¿Revisa las actualizaciones que Facebook hace a su política } \\
\text { de Datos? }\end{array}$ \\
\hline & & 4. ¿Sabe interpretar la política de privacidad de Facebook? \\
\hline & & $\begin{array}{c}\text { 5. ¿Conoce las acciones para control de información personal, } \\
\text { que Facebook le ha otorgado como usuario? }\end{array}$ \\
\hline & & $\begin{array}{l}\text { 6. ¿Sabe que la información personal que Facebook recopila de } \\
\text { los usuarios es compartida con alguien? }\end{array}$ \\
\hline & \multirow{4}{*}{$\begin{array}{c}\text { C. Nivel de } \\
\text { experticia en } \\
\text { las } \\
\text { configuracione } \\
\text { s de privacidad } \\
\text { disponibles en } \\
\text { la red social. }\end{array}$} & 1. ¿Crea Grupos de usuarios? \\
\hline & & $\begin{array}{c}\text { 2. ¿Conoce el efecto que tiene el seleccionar "Todos" en la } \\
\text { configuración de privacidad de Facebook? }\end{array}$ \\
\hline & & $\begin{array}{c}\text { 3. ¿Sabe cuál es la opción de configuración que un usuario } \\
\text { debería utilizar para restringir: quienes pueden enviarle } \\
\text { solicitudes de amistad; y, quienes pueden ver su lista de } \\
\text { amigos? }\end{array}$ \\
\hline & & $\begin{array}{l}\text { 4. ¿Sabe cuál es la opción de configuración que un usuario } \\
\text { debería utilizar para restringir en Facebook: la invitación de } \\
\text { alguien para asistir a un evento, las invitaciones a una app que } \\
\text { le haya enviado alguien, mensajes y vídeo-llamadas de } \\
\text { alguien, así como ponerse en contacto mediante la app de } \\
\text { Messenger? }\end{array}$ \\
\hline & D. Nivel de & 1. ¿Tiene conocimiento de donde Facebook guarda los datos \\
\hline
\end{tabular}




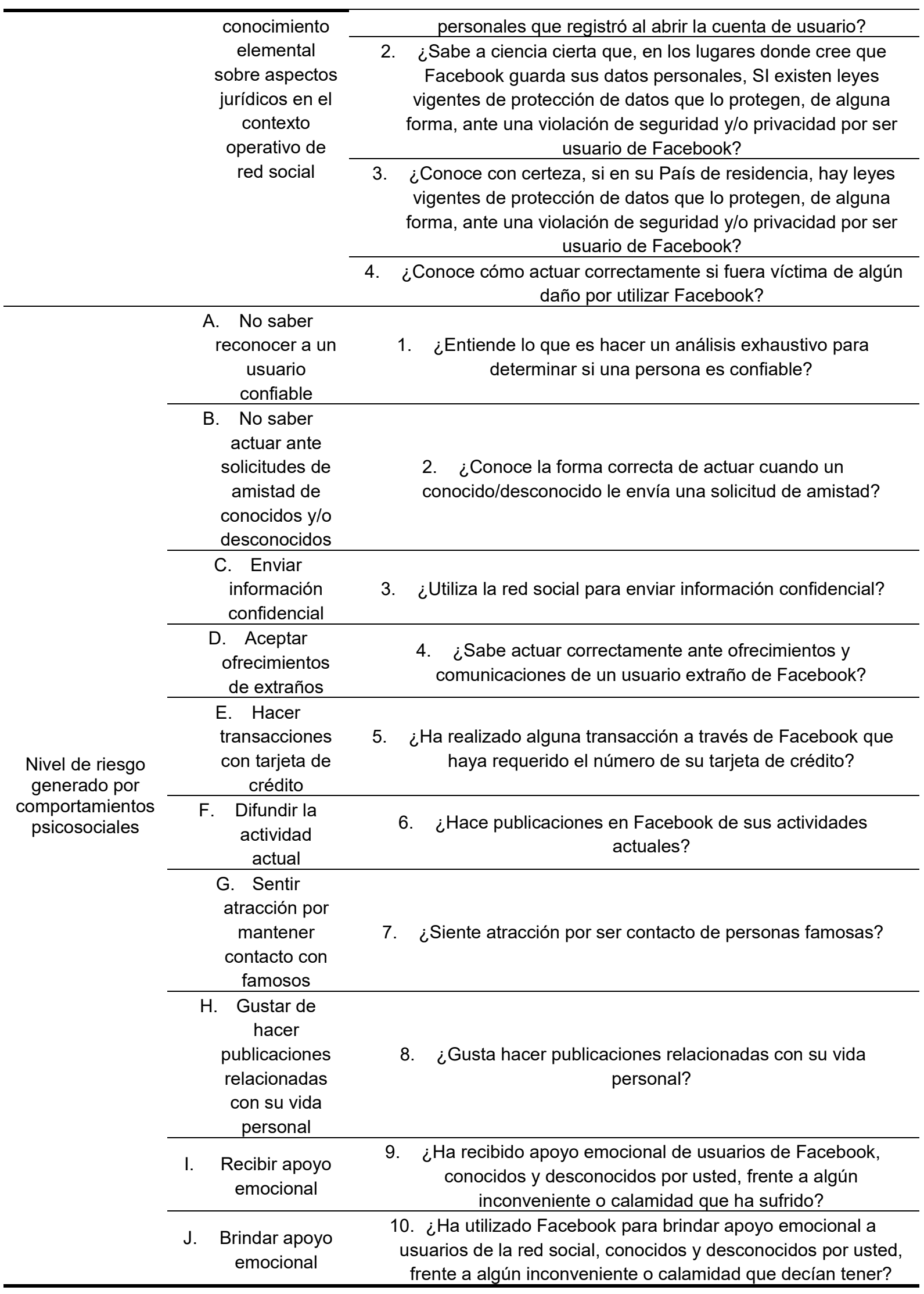




\begin{tabular}{cc} 
K. & $\begin{array}{c}\text { No clasificar a } \\
\text { sus contactos } \\
\text { en grupos } \\
\text { afines }\end{array}$ \\
\hline L. $\begin{array}{l}\text { Que Facebook } \\
\text { le aleje de sus } \\
\text { contactos cara } \\
\text { a cara }\end{array}$ & 11. ¿Clasifica a sus contactos en grupos afines? \\
& personas que convive a diario debido al uso de Facebook?
\end{tabular}

\subsection{Proceso Propuesto para la Estimación del Nivel Real de Conciencia de Usuarios de Facebook respecto a Privacidad y Seguridad en la Red Social}

EL proceso propuesto, Figura 4(a), inicia con la aplicación de la entrevista estructurada definitiva, obtenida con la depuración explicada en la sección marco metodológico, a la muestra de usuarios de Facebook en estudio. Cada una de las respuestas obtenidas deberán ser cuantificadas con un valor asignado según la respuesta; y con las respuestas a preguntas vinculadas se estructurarán los 32 atributos que definen el espacio multidimensional. Los atributos relacionados tendrán que ser agrupados para conformar 16 características significativas valoradas; y con ellas, se expresarán numéricamente los dos factores determinantes del grado de conciencia sobre privacidad y seguridad, que finalmente proveerán un valor al nivel real de conciencia de cada usuario de Facebook entrevistado, y con el cual se encasillará a la persona en uno de los 4 valores cualitativos, siguiendo lo descrito en la parte pertinente del marco metodológico. La aplicación del proceso propuesto a la muestra de usuarios ecuatorianos de Facebook, permitió obtener el resultado mostrado en la Figura 4(b).

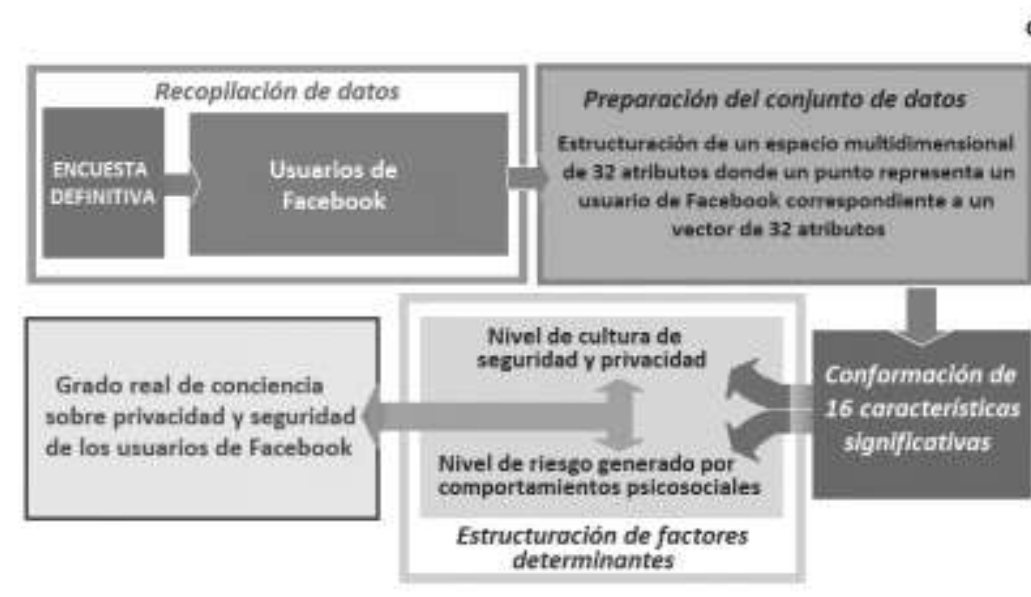

(a)
Grupo Etario Grado real de conciencioción

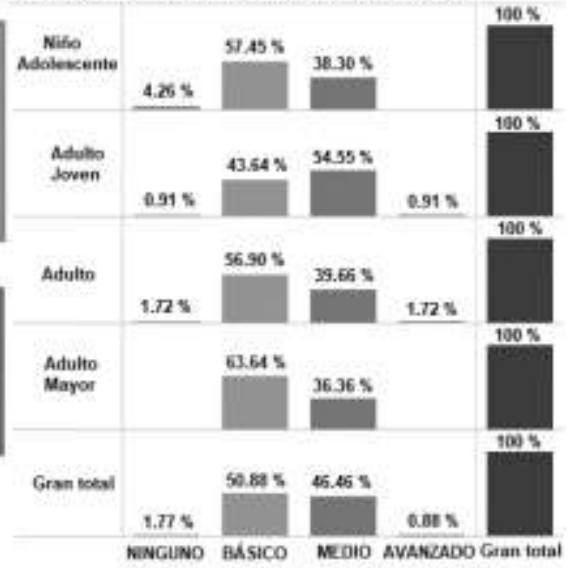

(b)

Figura 4. (a) Proceso propuesto para la estimación del nivel real de conciencia de usuarios de Facebook respecto a privacidad y seguridad en la red social - (b) Resultados obtenidos aplicando a la muestra el proceso propuesto 


\section{DISCUSIÓN}

El proceso desarrollado estima el nivel de conciencia sobre privacidad y seguridad de un usuario de Facebook, y lo posiciona en una de las cuatro instancias definidas: avanzado, medio, básico, o ninguno. La estimación reflejará fehacientemente la realidad del nivel de conciencia referido, solamente si la entrevista estructurada definitiva, planteada en este estudio, es respondida de una manera consciente y comprometida.
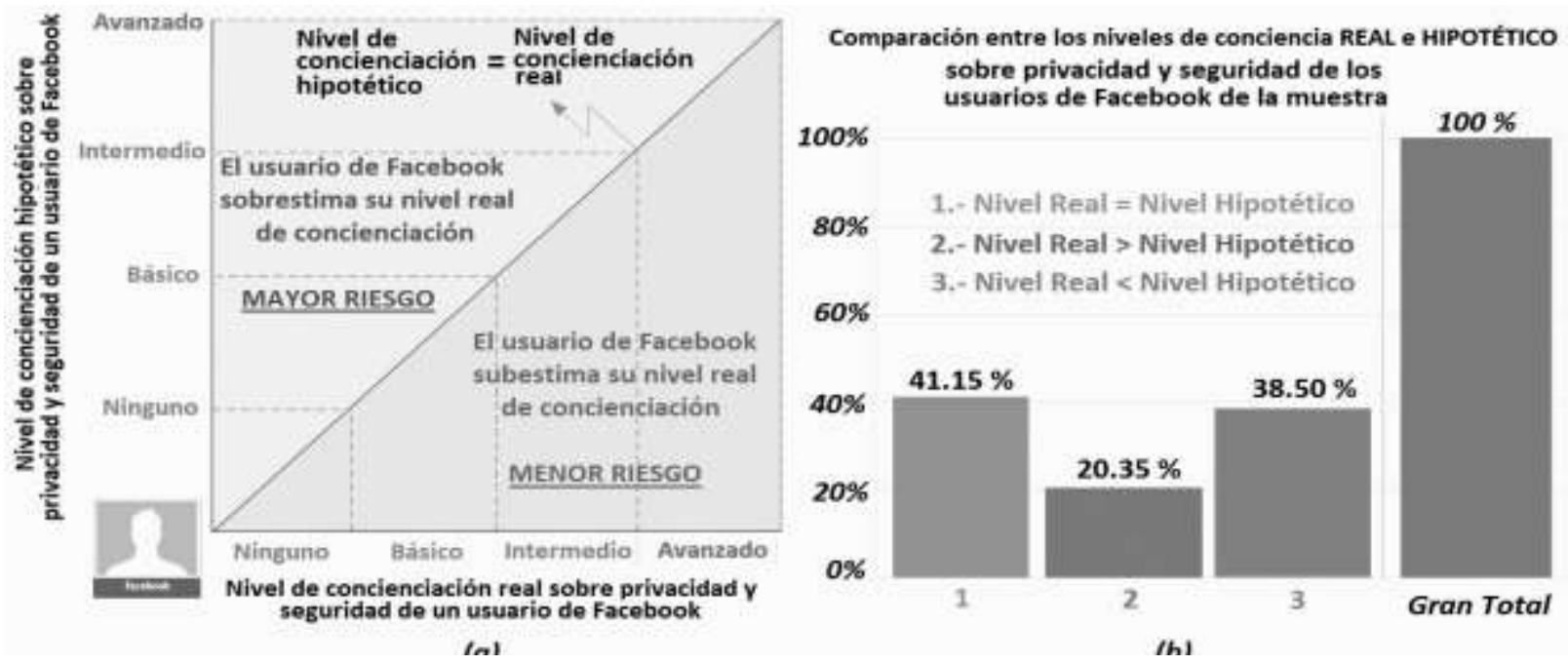

Figura 5. Comparación del nivel real de conciencia sobre privacidad y seguridad de un usuario de Facebook con su nivel hipotético: (a)Posibles ubicaciones para el usuario - (b) Resultados obtenidos de la muestra

Por otra parte, si se hace un análisis minucioso de los resultados intermedios que se van obteniendo en la tercera y cuarta etapas del proceso propuesto, es posible extraer otros aportes que complementan el conocimiento sobre nivel de conciencia sobre privacidad y seguridad, entre los que se pueden citar: 1) La comparación del nivel real de conciencia sobre privacidad y seguridad de un usuario de Facebook con el nivel hipotético de conciencia supuesto por él mismo, en la realidad permite situar a la persona en una de las dos alternativas siguientes, con respecto a su nivel real de conciencia sobre privacidad y seguridad en la red social: A) Hace una apreciación correcta, cualquiera que haya sido el nivel supuesto (nivel de conciencia hipotético = nivel de conciencia real); B) Hace una apreciación incorrecta, con dos posibles opciones: a) que el usuario se subestime (nivel de conciencia hipotético < nivel de conciencia real), b) que el usuario se sobrestime (nivel de conciencia hipotético > nivel de conciencia real), con las consecuencias sobre la exposición al riesgo que se representan en la Figura 5(a). Un resumen de los resultados obtenidos con 
la entrevista estructurada con respecto a este aporte complementario, se muestra en la Figura 5(b).

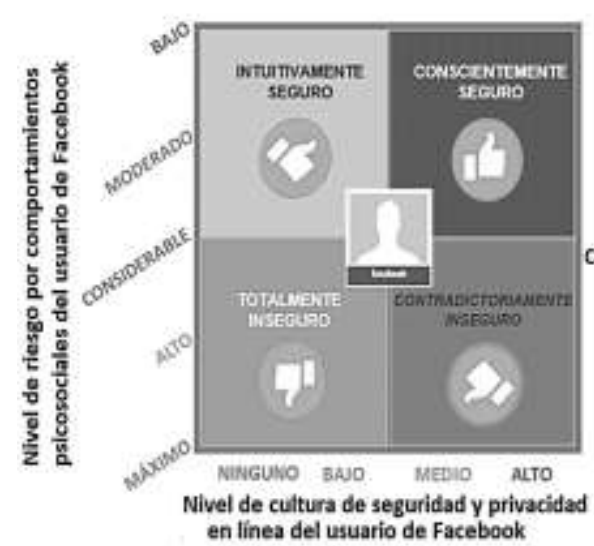

(a)

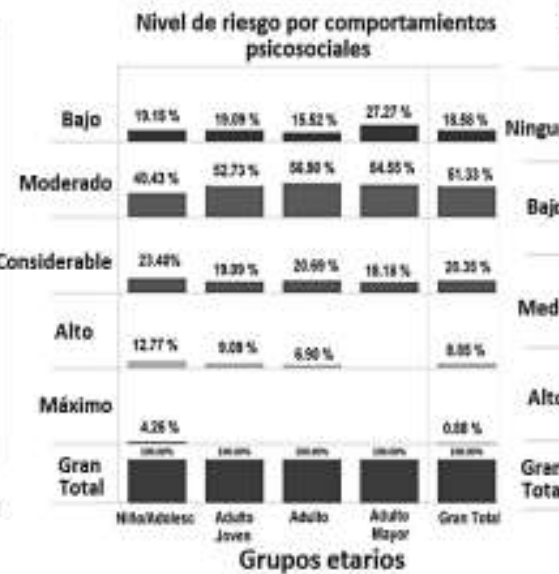

(b)
Nivel de cultura de seguridad y privacidad en linea del usuario de Facebook

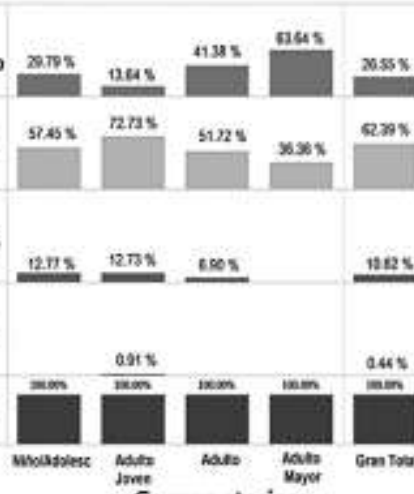

(c)

Figura 6. (a) Mapa de caracterización del Usuario de Facebook con relación a los factores determinantes del grado de conciencia sobre privacidad y seguridad. (b) Nivel de riesgo debido a comportamientos Psicosociales.

(c) Nivel de cultura de seguridad.

2) El posicionamiento de un usuario de Facebook, dentro del mapa de caracterización de la Figura 6 (a), de conformidad con la realidad en que desempeña sus interacciones virtuales a través de la red social; demostrando compatibilidad o incompatibilidad entre la forma natural, personal, y particular de actuar del usuario de Facebook al establecer sus relaciones interpersonales reales y virtuales (comportamientos psicosociales), y su nivel de cultura de seguridad y privacidad en línea. Resultados extraídos de la muestra relacionados con este aporte adicional se presentan en la Figura 6(b) y en la Figura 6(c). Este resultado complementario ayudaría a orientar acciones de mejoramiento para cada grupo de usuario particular, para fortalecer sus comportamientos psicosociales y/o su cultura de seguridad.

Se pueden llegar a identificar con mayor precisión los temas específicos de fortalecimiento dentro de cada uno de los factores determinantes; se presentan, como ejemplo, los resultados expuestos en las figuras: Figura 7(a), Figura 7(b), y Figura 7(c), que se corresponden respectivamente con los análisis porcentuales para los distintos grupos etarios respecto a: el nivel de conceptualización de los principios de seguridad y privacidad en línea, con el que se detecta que casi un tercio de la muestra, en todos los grupos etarios, requiere mejorar sus conceptualizaciones de seguridad y privacidad (niveles bajo y ninguno); el nivel de revisión de la política de datos, demuestra que el $98 \%$ de personas de la muestra no conoce como Facebook maneja los datos que recopila de los usuarios; y, el nivel de entendimiento de las distintas configuraciones de privacidad y seguridad disponibles en la 
red social, evidencia que el $73.45 \%$ de elementos de la muestra requiere perfeccionar este aspecto.

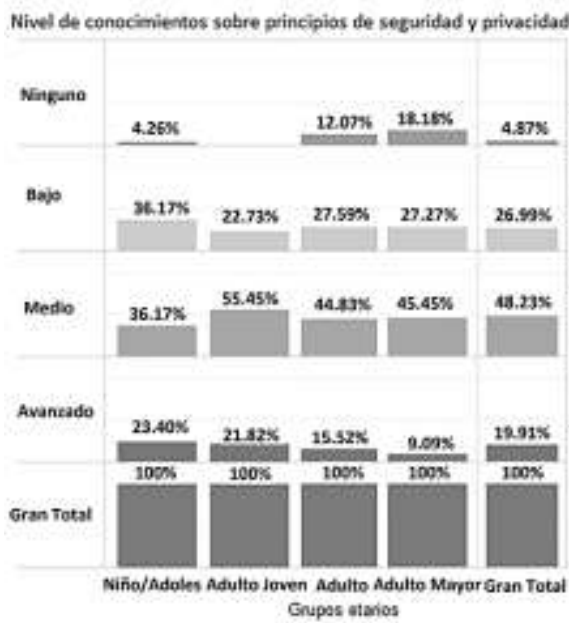

(a)

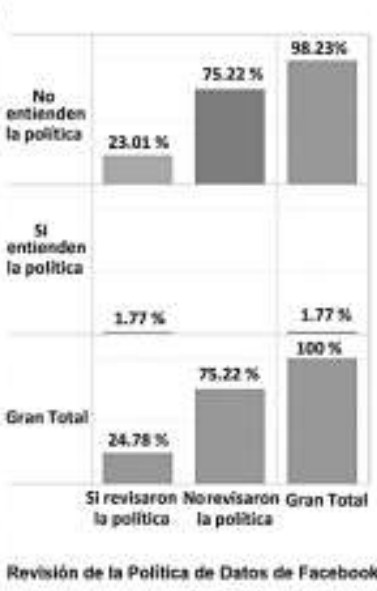

(b)

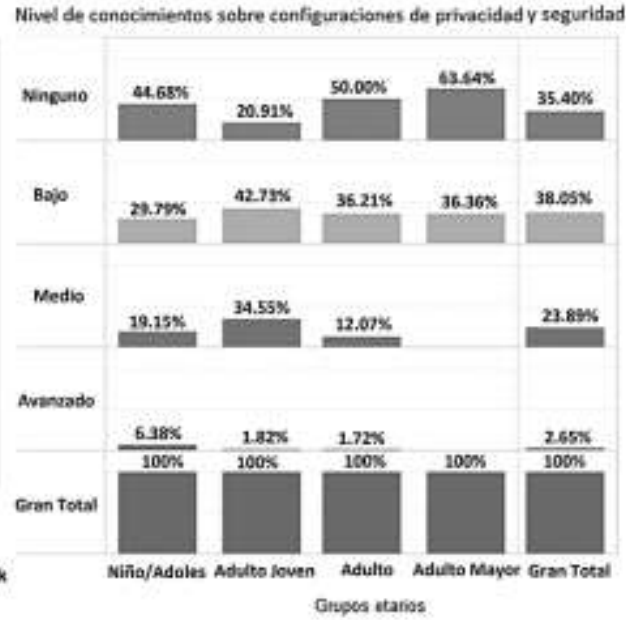

(c)

Figura 7. (a) Nivel de conceptualización de los principios de seguridad y privacidad en línea. (b) Nivel de revisión de la política de datos. (c) Nivel de entendimiento de las configuraciones de privacidad y seguridad.

\section{CONCLUSIONES}

Es posible afirmar que el proceso propuesto para estimar el nivel real de conciencia sobre privacidad y seguridad de un usuario de Facebook, provee datos válidos que reflejan con fidelidad la realidad.

La aplicación iterativa de los lineamientos dados por la Teoría Fundamentada y la hermenéutica, permitieron estructurar una sólida fundamentación, conformada por: 1) El modelo conceptual, con enfoque interdisciplinario, de las causas de los ciber-riesgos en el entorno de Facebook; y, 2) Los factores determinantes del nivel real de conciencia del usuario de Facebook respecto a privacidad y seguridad en la red social; instrumentos valiosos para comprender el proceso de estimación propuesto, y la interpretación de los resultados que se alcancen cuando el proceso sea aplicado.

El proceso planteado, es muy rico en datos complementarios que, interpretados correctamente, y combinados con el modelo conceptual interdisciplinario de las causas de los ciber-riesgos en el entorno de Facebook, ayudarían a plantear alternativas tendientes a mejorar los comportamientos psicosociales y la cultura de privacidad y seguridad del usuario de Facebook. Así por ejemplo, se concluye que el proveedor de la plataforma de la red social Facebook también puede impulsar el mejoramiento de la cultura de seguridad y 
privacidad de los usuarios, realizando ciertos ajustes en instancias de la interface de usuario, relacionadas con: 1) La creación de cuentas, de tal forma que un usuario sea registrado y dado de alta, solamente después de: A) evidenciar la revisión y la comprensión de la política de datos de Facebook, por ejemplo, respondiendo un sencillo cuestionario; y, B) la ejecución de una configuración básica de su privacidad. 2) La alerta de actualizaciones a las políticas de datos, para que el usuario acceda al servicio, posteriormente a una actualización de la política, solamente después de evidenciar la revisión de las modificaciones informadas. $Y, 3$ ) la presentación de la política de datos, para que el usuario cuente con las opciones necesarias para aceptar/autorizar las partes de la política con las que está de acuerdo, y rechazar las que se opongan a sus convicciones.

Finalmente se concluye que, para futuros estudios, es necesario incorporar en el modelo conceptual interdisciplinario de las causas de los ciber-riesgos para usuarios de Facebook, como parte del nivel de cultura de seguridad y privacidad, una vulnerabilidad adicional atribuible a los usuarios de Facebook: la tendencia de las personas a sobreestimar su nivel de conciencia sobre privacidad y seguridad en la red social.

\section{REFERENCIAS}

Algarni, A., Xu, Y., \& Chan, T. (2014). Social engineering in social networking sites: The art of impersonation. Proceedings - 2014 IEEE International Conference on Services Computing, SCC 2014, 797-804. https://doi.org/10.1109/SCC.2014.108

Cornejo, M., \& Tapia, M. L. (2011). Redes sociales y relaciones interpersonales en internet. Fundamentos En Humanidades, 2(24), 219-229. Retrieved from https://www.redalyc.org/articulo.oa?id=18426920010

Instituto de Auditores Internos de España. (2016). Ciberseguridad Una guía de supervisión. (Instituto de Auditores Internos de España, Ed.). Madrid. Retrieved from https://auditoresinternos.es/uploads/media_items/guia-supervision-ciberseguridad-fabrica-pensamientoiai.original.pdf

Lara, J. C., Pincheira, C., \& Vera, F. (2014). La privacidad en el sistema legal chileno. Retrieved from https://www.derechosdigitales.org/wp-content/uploads/pp-08.pdf

Me Conecto Sin Clavos, M. de G. de G. (2017). Guía ciber-riesgos educadores. Guatemala.

Netter, M., Herbst, S., \& Pernul, G. (2013). Interdisciplinary Impact Analysis of Privacy in Social Networks. In Y. Altshuler, Y. Elovici, A. B. Cremers, N. Aharony, \& A. Pentland (Eds.), Security and Privacy in Social Networks (pp. 1-253). Springer. https://doi.org/10.1007/978-1-4614-4139-7

OECD. (1980). Annex to the recommendation of the Council of 23 September 1980: Guidelines governing the protection of privacy and transborder flows of personal data.

OECD. (2011). Thirty Years After the Oecd Privacy Guidelines. Retrieved from http://www.oecd.org/sti/ieconomy/49710223.pdf

UIT, U. I. T. (2014). Manual para la medición del uso y el acceso a las TIC por los hogares y las personas 
(2014th ed.). Retrieved from http://www.itu.int/dms_pub/itu-d/opb/ind/D-IND-ITCMEAS-2014-PDF-S.pdf

UNESCO. (2012). Global Survey on Internet Privacy and Freedom of Expression. UNESCO series on Internet freedom. https://doi.org/ISBN: 978-92-3-104241-6

Wallbridge, R. (2009). How safe is Your Facebook Profile? Privacy issues of online social networks. ANU Undergraduate Research Journal, 1, 85-92. Retrieved from http://pressfiles.anu.edu.au/downloads/press/n1677/pdf/11.pdf

Wong, K. (2014). Trust and Privacy Exploitation in Online Social Networks, (October), 28-33. https://doi.org/10.1109/MITP.2009.1.

Zhan, J., \& Fang, X. (2011). Social computing: the state of the art. Journal of Social Computing and CyberPhysical Systems, 1(1), 12. Retrieved from http://www.inderscienceonline.com/doi/abs/10.1504/IJSCCPS.2011.043601

Zhang, C., Sun, J., Zhu, X., \& Fang, Y. (2010). Privacy and Security for Online Social Networks: Challenges and Opportunities. IEEE Network, 24(4), 13-18. https://doi.org/10.1109/MNET.2010.5510913 